\title{
Southern infrared proper motion survey
}

\section{Discovery of new high proper motion stars from first full hemisphere scan}

\author{
N. R. Deacon, N. C. Hambly, and J. A. Cooke
}

Institute for Astronomy, University of Edinburgh, Blackford Hill, Edinburgh EH9 3HJ, UK

e-mail: [nd;n.hambly; j. cooke] @roe.ac.uk

Received 13 September 2004 / Accepted 30 November 2004

\begin{abstract}
We present the first results from the Southern Infrared Proper Motion Survey. Using 2 Micron All Sky Survey data along with that of the SuperCOSMOS sky survey we have been able to produce the first widefield infrared proper motion survey. Having targeted the survey to identify nearby M, L and T dwarfs we have discovered 70 such new objects with proper motions greater than $0.5^{\prime \prime} / \mathrm{yr}$ with 10 of these having proper motions in excess of $1^{\prime \prime} / \mathrm{yr}$. The most interesting of these objects is SIPS1259-4336 a late M dwarf. We have calculated a trigonometric parallax for this object of $\pi=276 \pm 41$ milliarcsec yielding a distance of $3.62 \pm 0.54 \mathrm{pc}$. We have also discovered a common proper motion triple system and an object with a common proper motion with LHS 128. The survey completeness is limited by the small epoch differences between many 2MASS and UKI observations. Hence we only recover $22 \%$ of Luyten objects with favourable photometry. However the Luyten study is itself unquantifiably incomplete. We discuss the prospect of enhancing the survey volume by reducing the lower proper motion limit.
\end{abstract}

Key words. astrometry - stars: low mass, brown dwarfs

\section{Introduction}

The Solar Neighbourhood provides an opportunity to constrain the field mass and luminosity functions of Low Mass Stars and Brown Dwarfs. As Low Mass Stars and Brown Dwarfs represent the products of star formation they provide clues as to the processes involved. They also represent a sink for baryonic matter and hence may explain a small proportion of Galactic dark matter. In addition nearby multiples can give clues about the formation of such systems.

Proper motion surveys are a useful tool for discovering nearby stars. The benchmark for such surveys is Luyten's Half Arcsecond Catalogue (hereafter LHS) (1979) containing 3587 stars with $\mu \geq 0.5^{\prime \prime} / \mathrm{yr}$. This survey is incomplete in the southern sky and excludes fields in the galactic plane. Modern high proper motion searches seek to complete Luyten's work in the southern sky and to identify objects Luyten failed to find. Some of these use manual blinking techniques such as the Calan-ESO proper motion catalogue (Ruiz et al. 2001) and the study of Wroblewski \& Costa (2001). Recent computational methods include those of Pokorny et al. (2003) and Scholz et al. (2002). Pokorny lists 6206 stars with $\mu \geq 0.18^{\prime \prime} / \mathrm{yr}$ found using SuperCOSMOS scans of 131 Schmidt fields. Scholz found 15 stars with high proper motions from APM scans of UKST plates. Eight of these have distances less than $25 \mathrm{pc}$. Lepine \& Shara (2002) have used POSS plates to search in the

* Tables 5 and 6 are only available in electronic form at the CDS via anonymous ftp to cdsarc.u-strasbg.fr $(130.79 .128 .5)$ or via http://cdsweb.u-strasbg.fr/cgi-bin/qcat?J/A+A/435/363 northern hemisphere. This has been done with the SUPERBLINK algorithm, in which plates from two different epochs (POSS I and POSS II) are aligned and the later, better POSS II plates degraded to make them of comparable quality to the POSS I plates. The two images are then subtracted making the moving, high proper motion objects obvious. Teegarden et al.'s (2003) recent discovery was made using data from the SkyMorph search for near earth objects, which also picks up stars with a very high proper motion. Most recently Hambly et al. (2004) have used SuperCOSMOS data to identify five new high proper motion stars south of $\delta=-60^{\circ}$.

However the majority of these surveys utilise optical $R$ plate data and many nearby stars are so red that they are barely visible on such plates. Low Mass Stars and Brown Dwarfs are bright in the near infrared $I, J, H$ and $K_{\mathrm{S}}$. This means that a proper motion search in the near infrared is ideal for identifying such objects. Near infrared proper motion surveys have already been used to discover low mass companions to nearby stars (Scholz et al. 2004) and to confirm that companions are co-moving with the primary (Seifahrt et al. 2004). However these are targeted at known nearby stars and are not useful for discovering new systems. Many proper motion surveys are also incomplete. SCR1845-6357 for example was missed by all surveys prior to Hambly et al. (2004) despite being relatively bright at $R \approx 16$ and having a high proper motion $\mu>2^{\prime \prime} / \mathrm{yr}$. It is likely that similar objects are still to be discovered. In order to identify late $\mathrm{M}, \mathrm{L}$ and $\mathrm{T}$ dwarfs a proper motion survey in the near infrared is required. For our survey we used 
SuperCOSMOS $I$ data and data in $J, H, K_{\mathrm{S}}$ from the 2MASS Point Source Catalogue as our two epochs. 2MASS objects likely to be nearby low mass stars or brown dwarfs were selected. These were then paired with likely $I$ plate counterparts. This constitutes the first widefield infrared proper motion survey ideal for identifying nearby Low Mass Stars and Brown Dwarfs. Here we present details of the method used as well as a list of objects found with $\mu>0.5^{\prime \prime} / \mathrm{yr}$.

\section{Candidate identification}

\subsection{Initial 2MASS object selection}

Firstly candidate 2MASS images had to be selected. To ensure that these objects were not too close to the photometric limit of the 2MASS survey, the objects had to be brighter than $J=16$. Elliptical objects may have inaccurate photometry and astrometry, hence selected objects had to have an axial ratio less than 1.4. Crowded regions near the galactic plane will produce many spurious detections of high proper motion objects. In order to reduce this crowding any object with $|b|<15^{\circ}$ was excluded. The 2MASS Point Source Catalogue includes a parameter indicating the proximity of the nearest source; the Executive Summary for the catalogue states that any object closer than $6^{\prime \prime}$ to its nearest neighbouring source must be treated with caution. Hence such objects were excluded. Objects categorised by the 2MASS survey as being associated with extended sources or minor planets were also removed.

Objects which met these criteria were then subjected to a series of colour cuts.

In order to make the process as efficient as possible, only objects whose photometry suggested that they were M, L and $\mathrm{T}$ dwarfs were selected. In order to do this regions on a colourcolour diagram were marked out as likely to contain objects of a particular spectral type. Figure 1a shows these regions and Fig. 1b shows the colours of objects taken from various surveys. Note that because there is an overlap between the mid M and mid $\mathrm{T}$ spectral classes on a colour-colour diagram any object that falls in this region is treated as if it could be either spectral type. Several early M dwarfs fall outside the bounds of the $\mathrm{M}$ dwarf region. When the full survey is produced this will have to be included in completeness estimates. Many T dwarfs fall outside the $\mathrm{T}$ dwarf region, this scatter is due to photometric errors. As we have set an $I$ plate limit and an $I-J$ cut we will only identify the brightest $\mathrm{T}$ dwarfs. Dimmer objects have higher photometric errors and hence higher scatter. As these objects are already excluded due to our other photometric cuts it does not matter if they fall outside the $\mathrm{T}$ dwarf region on the colour colour diagram.

Once objects have passed the colour cuts and been categorised it is then necessary to find if they are in fact moving with significant proper motions. Figure 2 shows the relative positional errors between SuperCOSMOS and 2MASS data. These were calculated by finding the positional shift of a set of 2MASS objects and calculating the mean error using a method of median absolute deviation. On the basis of these results it was decided to set one arcsecond (roughly $4-5 \sigma$ ) as the lower "movement cut". This ensures that only objects which appear (a)

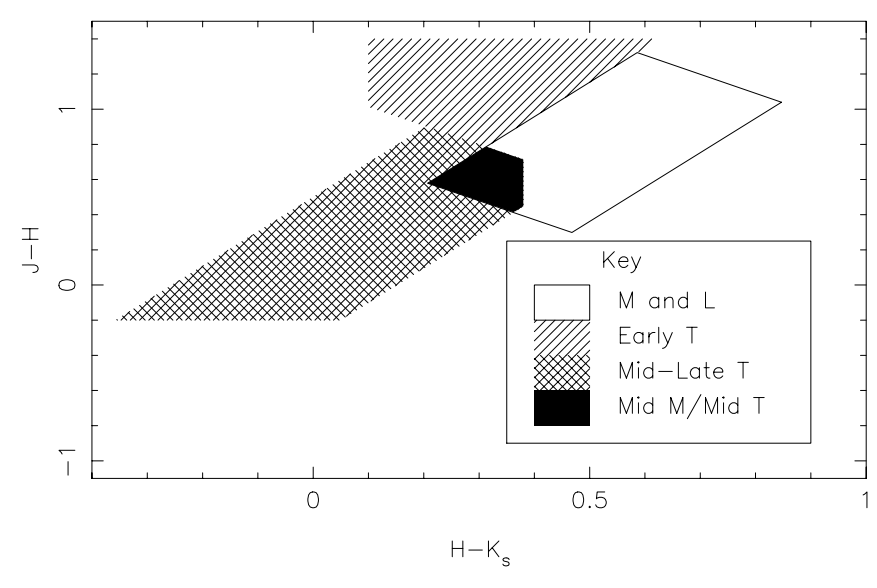

(b)

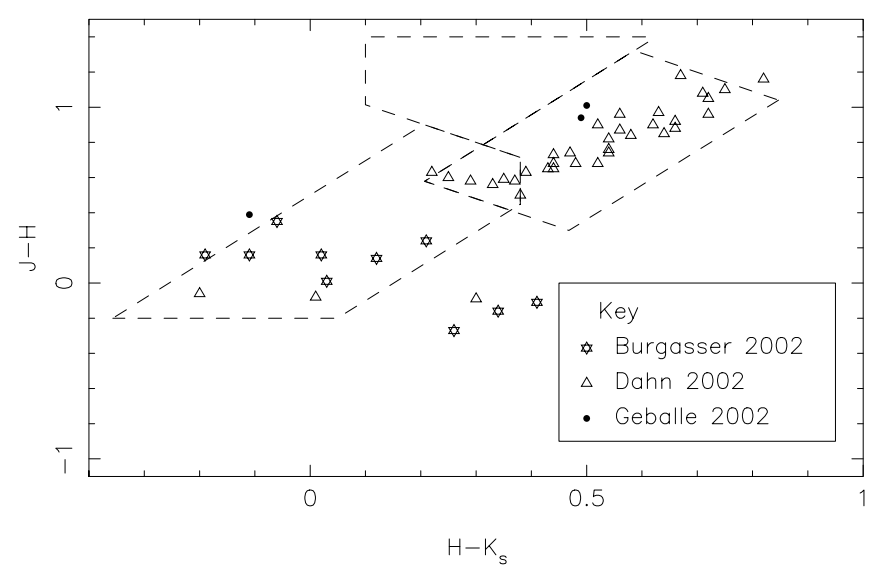

Fig. 1. The regions in the colour-colour diagram within which objects were deemed to be M, L or T dwarfs.

to have moved from one epoch to the other are included in the sample. Any 2MASS object found to have an $I$ plate counterpart within one arcsecond was discounted as not having a significant enough proper motion. This cut has implications for proper motion completeness which will have to be taken into account. Also to remove spurious detections due to bright stellar halos, any 2MASS object within $10^{\prime \prime}$ of an I plate image flagged as being close to a bright star was removed. Images blended on the $I$ plates can cause positional offsets, hence any 2MASS object within $10^{\prime \prime}$ of a bright $(I<14)$ highly elliptical object or 6 " of one deblended by SuperCOSMOS software was removed.

\subsection{Pairing with potential I plate counterparts}

Once it is established that objects have appeared to move their I plate counterparts need to be identified. In order to do this a maximum proper motion of $\mu_{\max }=10^{\prime \prime} / \mathrm{yr}$ was set meaning only $I$ plate objects within a maximum radius $r_{\max }=\mu_{\max } \Delta t$ of the 2MASS object would be paired, where $\Delta t$ is the time elapsed between the two observations. This maximum proper motion was chosen to be roughly equal to that of Barnard's star, the star with the highest known proper motion, this allows us to 
(a)

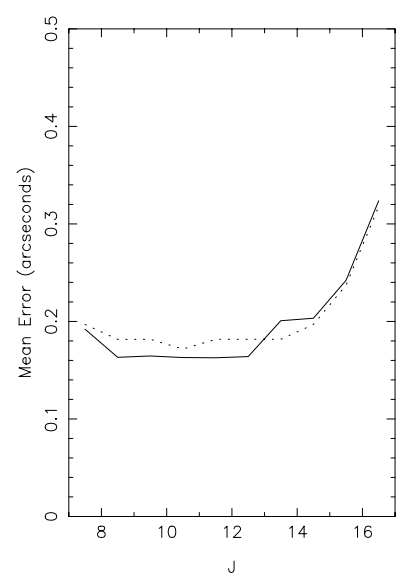

(c)

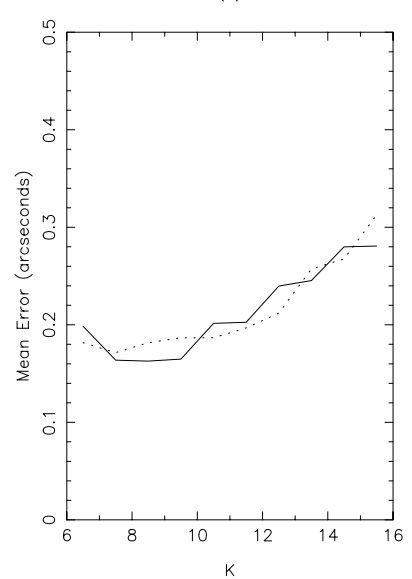

(b)

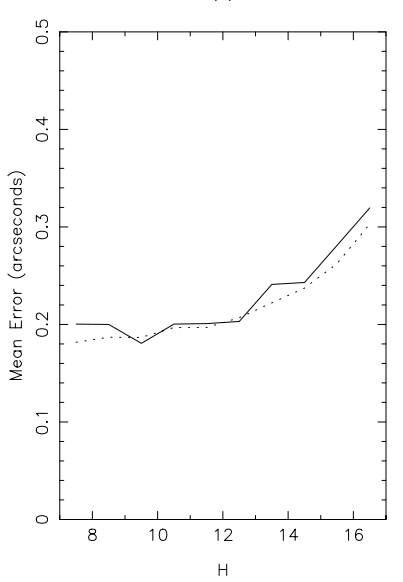

The I plate images also had to pass tests to indicate that they were good stellar images. Objects deblended by the SuperCOSMOS software were excluded as it is difficult to gather accurate astrometric information from them. Images with axial ratios higher than 1.7 were also excluded as they may have poor astrometry or may be blended objects which have not been separated by the software. I plate counterpart objects also had to be stellar in nature and not be in close proximity to bright stars.

Candidates for $I$ plate counterpart images also had to pass a series of photometric tests. Firstly to ensure that the $I$ plate counterparts were red enough to be true Low Mass Stars or Brown Dwarfs any object which had a UKST $R$ plate magnitude had to pass the cut $(R-I)>2$. As noted in Sect. 2.1, 2MASS objects were classified as being, M and L dwarfs, early $\mathrm{T}$ dwarfs, mid-late $\mathrm{T}$ Dwarfs or as lying in the overlap region on the colour-colour diagram between $\mathrm{M}$ dwarfs and mid-T Dwarfs. Objects classified as $\mathrm{M}$ and $\mathrm{L}$ dwarfs had to fulfill the condition $1.0<I-J<4.8$. Objects classified as early $\mathrm{T}$ Dwarfs or mid-late $\mathrm{T}$ dwarfs had to be within the range $4.0<I-J<5.5$. Any object falling in the overlap region between $\mathrm{M}$ dwarfs and mid-T dwarfs could be of either type so had to conform to the conservative cut of $1.0<I-J<5.5$

In order to ensure that the $I$ plate images selected were true counterparts and not unrelated objects two additional processes were carried out. Firstly it was checked that the $I$ plate image was not associated with (within one arcsecond of) another 2MASS image. Secondly inspection by eye of the images ensured that only true high proper motion objects were selected.

\section{Determination of proper motions}

Fig. 2. The relative positional errors between SuperCOSMOS and 2MASS. The solid line represents the errors in Right Ascension while the dotted line shows those in Declination.

identify very high proper motion stars without being swamped by spurious pairings. Once an I plate object was identified as within $r_{\max }$ of the target it had to pass a series of tests to prove it was indeed a plausible counterpart to the 2MASS object.

Firstly the $I$ plate object had to have astrometry suggesting it was a high proper motion star. These data come from the calculated astrometric solutions in the SuperCOSMOS sky survey (Hambly et al. 2001). Any I plate object with an astrometric solution that places it at the same position (within the proper motion errors quoted in the astrometric solution) as the 2MASS object at the epoch of the 2MASS observation will be included as a possible counterpart. Many objects will either be so red that they will only appear on the $I$ plates also objects moving with high proper motions will have moved too far to be paired in other bands so will be recorded as only being on the $I$ plates. Hence any object appearing only on the $I$ plates was included as a possible counterpart. Finally some $I$ plate objects will be incorrectly paired with unrelated objects on the $B_{J}$ or $R$ plates and hence will have an inaccurate astrometric solution. Such objects will have a high value of $\chi_{v}^{2}\left(\chi^{2}\right.$ per degree of freedom), hence any object with $\chi_{v}^{2}>2.0$ was included as a possible counterpart.

Objects in this paper are initially selected using proper motions calculated by finding the distance an object had moved between the two epochs and dividing by the time elapsed. Any object with such a calculated proper motion greater than $0.4^{\prime \prime} / \mathrm{yr}$ was selected to have its proper motion more accurately calculated. The SuperCOSMOS Sky Survey software calculates proper motions using $B_{J}, I$ and two epoch $R$ measurements for each object in the database. Hence, in theory, we should have access to astrometric solutions for all the objects selected so far. Unfortunately many do not have suitable solutions, stars with very high proper motions will have large shifts in their positions from plate to plate meaning they will often be too far apart from one epoch to the next for the the SSS software to identify them as the same object. Hence such stars will not have calculated proper motions. Some high proper motion objects may be spuriously paired with faint unrelated objects on one or more plates, producing an unreliable astrometric solution. Also the reddest objects may only appear on the $I$ plate and hence will have no calculated astrometric solution. In order to make sure that all objects had a reliable astrometric solution a procedure to calculate the astrometric solution using only I plate and 2MASS data has been developed. Firstly a set of reference stars with $I$ and $J$ magnitudes similar to the star whose proper motion we wish to calculate was selected. These reference stars are then used to produce a linear fit between the plates, to account for plate to plate scaling and 


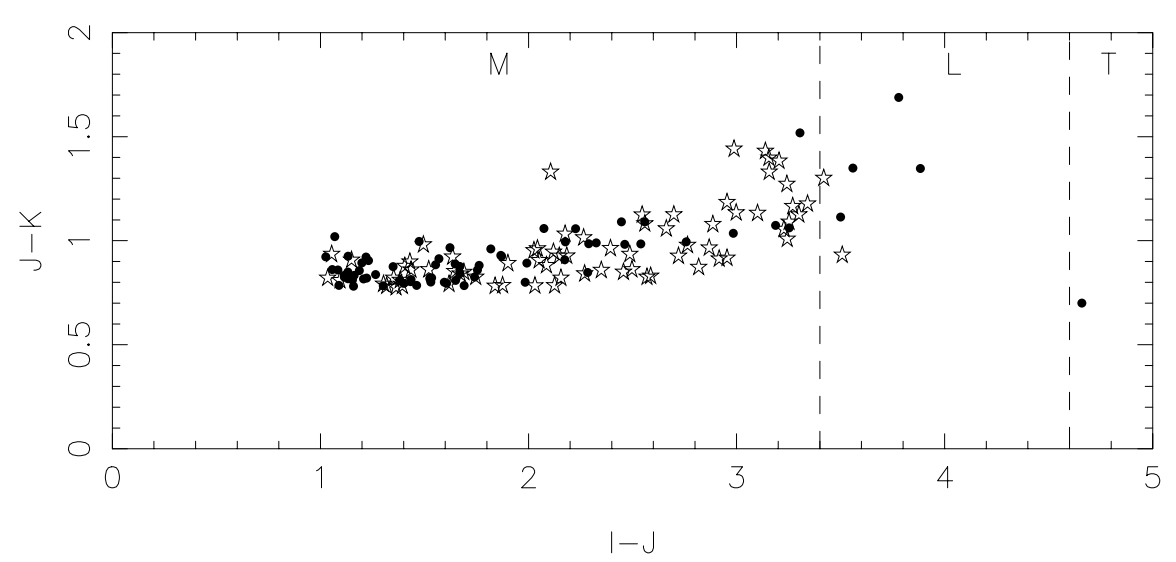

Fig. 3. A two colour diagram for all the objects found in the survey. The star symbols represent new discoveries while the solid circles are previously known objects.

Table 1. Schmidt photographs used in this study; relative astrometric quality is indicated (see text). One plate was excluded due to poor astrometric quality.

\begin{tabular}{|c|c|c|c|c|c|c|c|c|c|}
\hline $\begin{array}{l}\text { Plate } \\
\text { No. }\end{array}$ & $\begin{array}{c}\text { Date } \\
(\mathrm{dd} / \mathrm{mm} / \mathrm{yy})\end{array}$ & "LST & $\begin{array}{c}\text { Zenith } \\
\text { angle }\end{array}$ & $\begin{array}{l}\text { Emul- } \\
\text { sion }\end{array}$ & Filter & $\begin{array}{l}\text { Exp. } \\
(\min )\end{array}$ & $\begin{array}{c}\sigma_{x} \\
(\mathrm{mas})\end{array}$ & $\begin{array}{c}\sigma_{y} \\
(\mathrm{mas})\end{array}$ & Material \\
\hline \multicolumn{10}{|c|}{ Plates Used } \\
\hline R 5108 & $25 / 06 / 79$ & 1254 & $12.5^{\circ}$ & IIIaF & RG 630 & 800 & 64 & 65 & $\begin{array}{l}\text { Original non-survey } \\
\text { plate }\end{array}$ \\
\hline ESOR4809 & $19 / 05 / 82$ & 1200 & $18.6^{\circ}$ & IIIaF & RG 630 & 120 & 63 & 49 & $\begin{array}{l}\text { Copy of ESO survey } \\
\text { plate }\end{array}$ \\
\hline OR14770 & $12 / 02 / 92$ & 1245 & $12.6^{\circ}$ & IIIaF & OG 590 & 600 & 41 & 49 & $\begin{array}{l}\text { Original survey } \\
\text { plate }\end{array}$ \\
\hline OR16928 & $26 / 01 / 96$ & 1142 & $19.6^{\circ}$ & 4415 & OG 590 & 150 & 68 & 67 & $\begin{array}{l}\text { Original non-survey } \\
\text { film }\end{array}$ \\
\hline OR17069 & $26 / 04 / 96$ & 1322 & $13.1^{\circ}$ & $\mathrm{IIIaF}$ & OG 590 & 100 & 56 & 55 & $\begin{array}{l}\text { Original non-survey } \\
\text { plate }\end{array}$ \\
\hline OR17997 & $28 / 04 / 98$ & 1314 & $12.7^{\circ}$ & IIIaF & OG 590 & 600 & 77 & 65 & $\begin{array}{l}\text { Original non-survey } \\
\text { plate }\end{array}$ \\
\hline
\end{tabular}

orientation errors. The stars positions are then corrected for plate to plate errors and used to find the proper motion. The plate to plate fit also provides an estimate of the typical error for objects near the target star and this is used to calculate the error in the proper motion. In the case where an object has a calculated astrometric solution both from the I plate-2MASS fitting procedure and from the SuperCOSMOS software the most accurate one is quoted. Unfortunately some objects did not have enough reference stars to produce a plate model. These had their proper motions found in a same way to those with enough reference stars with the only difference being the lack of corrections for plate to plate errors. The error estimate for these stars was calculated using the positional errors shown in Fig. 2.

\section{Results}

Table 5 contains the astrometric data for all the objects identified with proper motions greater than $0.5^{\prime \prime} / \mathrm{yr}$. There are 70 new objects and 73 objects identified by previous surveys. The $I, J, H$ and $K_{\mathrm{s}}$ photometry for each object are also listed in Table 6. Figure 3 shows the colours of the newly discovered objects. The reddest newly discovered object is SIPS0641-4322 which has colours suggesting it is an early L dwarf. Objects such as this would be difficult to find using purely optical proper motion surveys. Figure 4 shows histogram of the number of newly discovered and previously known objects vs. magnitude $(J)$ and colour $\left(I_{J}\right)$. It is clear that the newly discovered objects are generally fainter and redder than those previously known. Below we present notes on the most interesting individual newly identified objects identified.

\subsection{Notes on individual objects}

\subsubsection{SIPS1259-4336}

Infrared colours indicate this object is an M 8-9 dwarf 2000. It is one magnitude fainter than the similar object SCR1845-6357 2004 placing it at a distance of roughly 6pc. In addition its high proper motion suggests that it is a nearby star. Hence as there were several non-survey plates available for this object in the UKST plate library a trigonometric parallax measurement was carried out. This draws on techniques used in Deacon \& Hambly (2001) and Deacon et al. (2005). Four of the non-survey plates were selected for their good parallax factors and were scanned on SuperCOSMOS 
New Discoveries

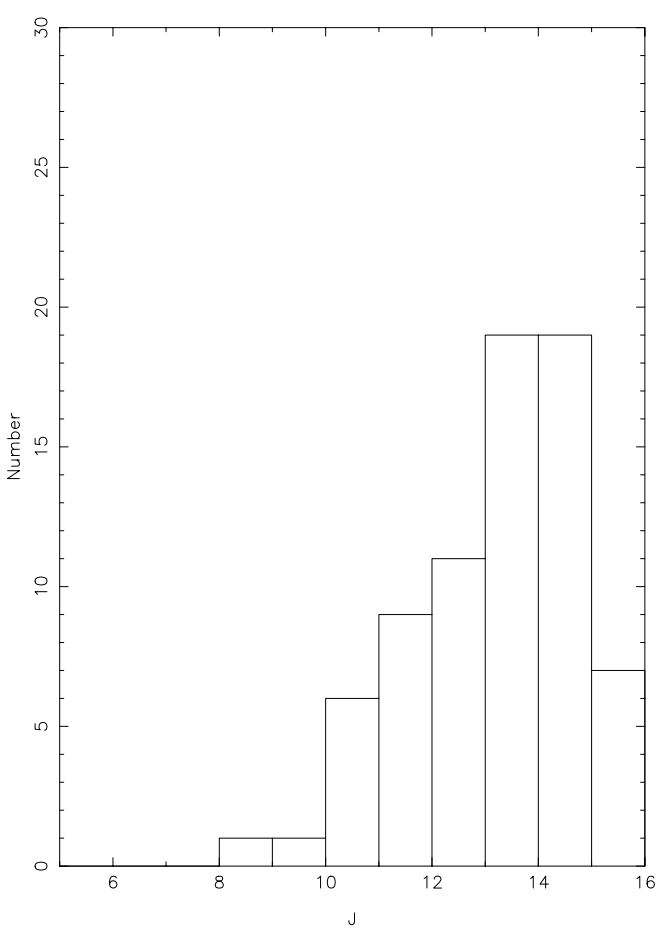

Previously known objects

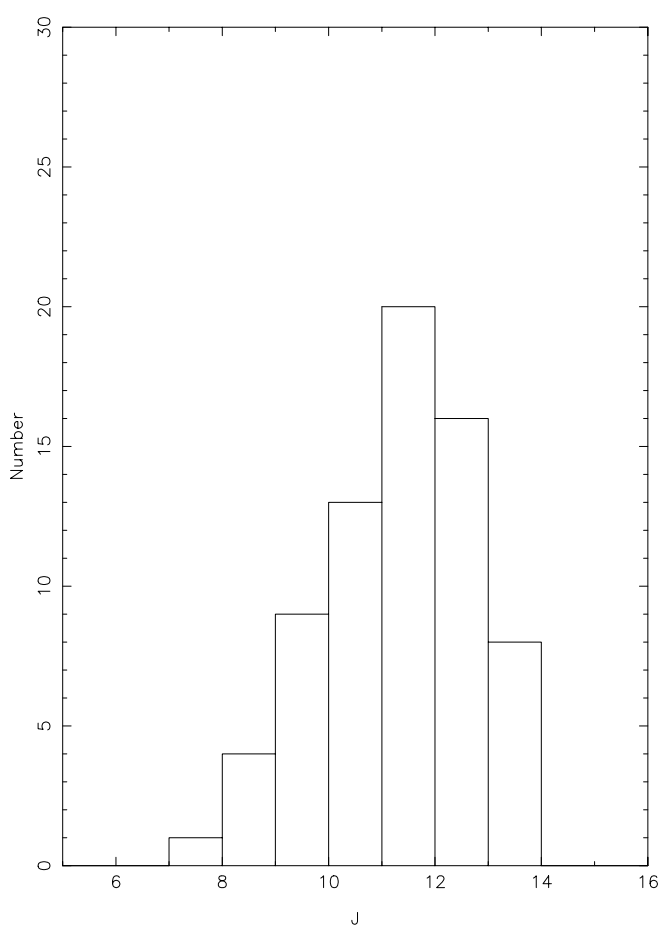

New Discoveries

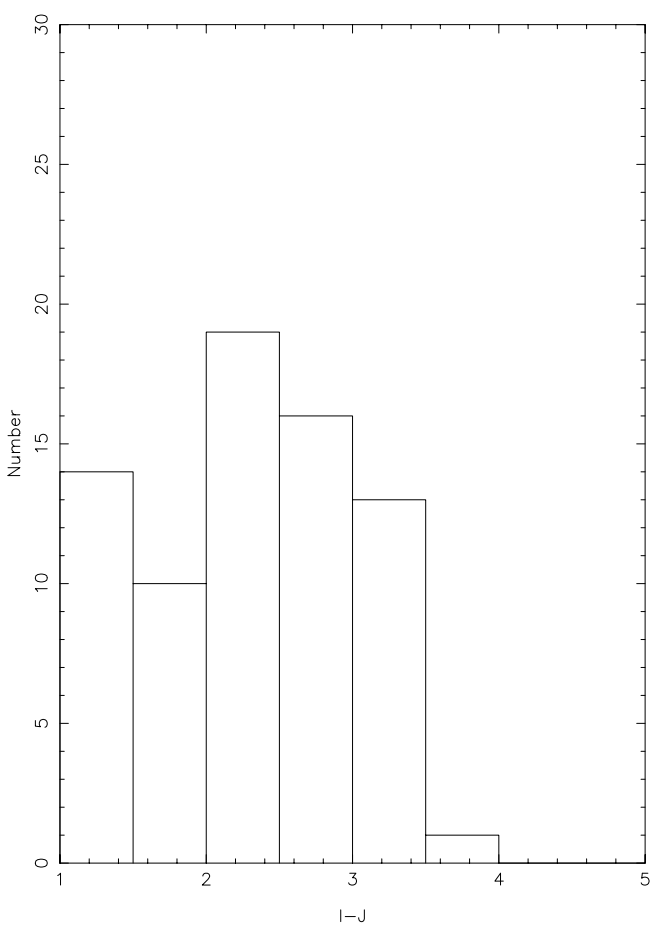

Previously known objects

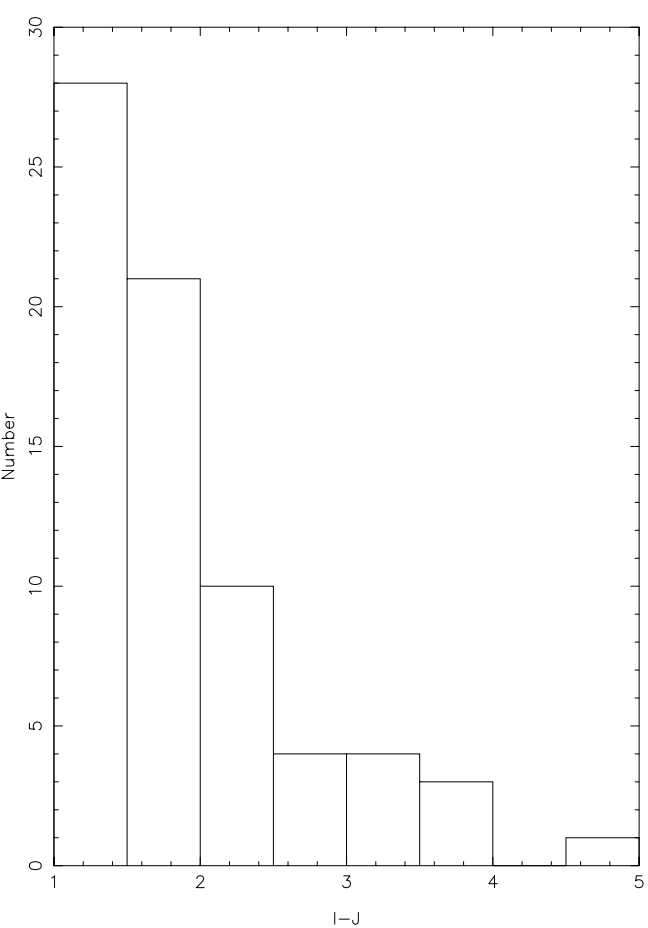

Fig. 4. Histograms showing the magnitude $(J)$ and colour $(I-J)$ of both the newly discovered and previously discovered objects.

and used along with the existing UKST R and ESO R plates. Details of the plates used are shown in Table 1.

The data from these plates were reduced using the same method as SCR1845-6357. A total of 148 reference stars were used to produce the plate to plate models. Once these had been applied to correct for plate to plate errors the astrometric solution could be found, this is shown in Table 2. The deviation from the proper motion is shown in Fig. 6 with the parallax ellipse traced out by the object shown in Fig. 7. To check for any systematic errors the reference stars were run through the fitting procedure. Their proper motions and parallaxes are show in Fig. 8 along with those for SIPS1259-4336. It is clear that there is no significant offset in the parallax and that SIPS12594336 is well separated from the mass of reference stars. The mean $\chi_{v}^{2}$ for the reference stars was found to be 0.97 indicating good model fits. Spectroscopic data for this object were 
Table 2. The full astrometric solution for SIPS1259-4336.

\begin{tabular}{cccl}
\hline \hline Parameter & Fitted value & Error & Units \\
\hline RA on 01/01/2000 & $12^{\mathrm{h}} 59^{\mathrm{m}} 04^{\mathrm{s}} .760$ & 0.047 as & - \\
Dec on 01/01/2000 & $-43^{\circ} 36^{\prime} 24^{\prime \prime} 21$ & 0.047 as & - \\
$\mu_{\alpha}$ & 1.105 & 0.004 & $\mathrm{as} / \mathrm{yr}$ \\
$\mu_{\delta}$ & -0.262 & 0.004 & $\mathrm{as} / \mathrm{yr}$ \\
$\pi$ & 276 & 41 & $\mathrm{mas}$ \\
$\chi_{v}^{2}$ & 0.65 & & \\
\hline
\end{tabular}

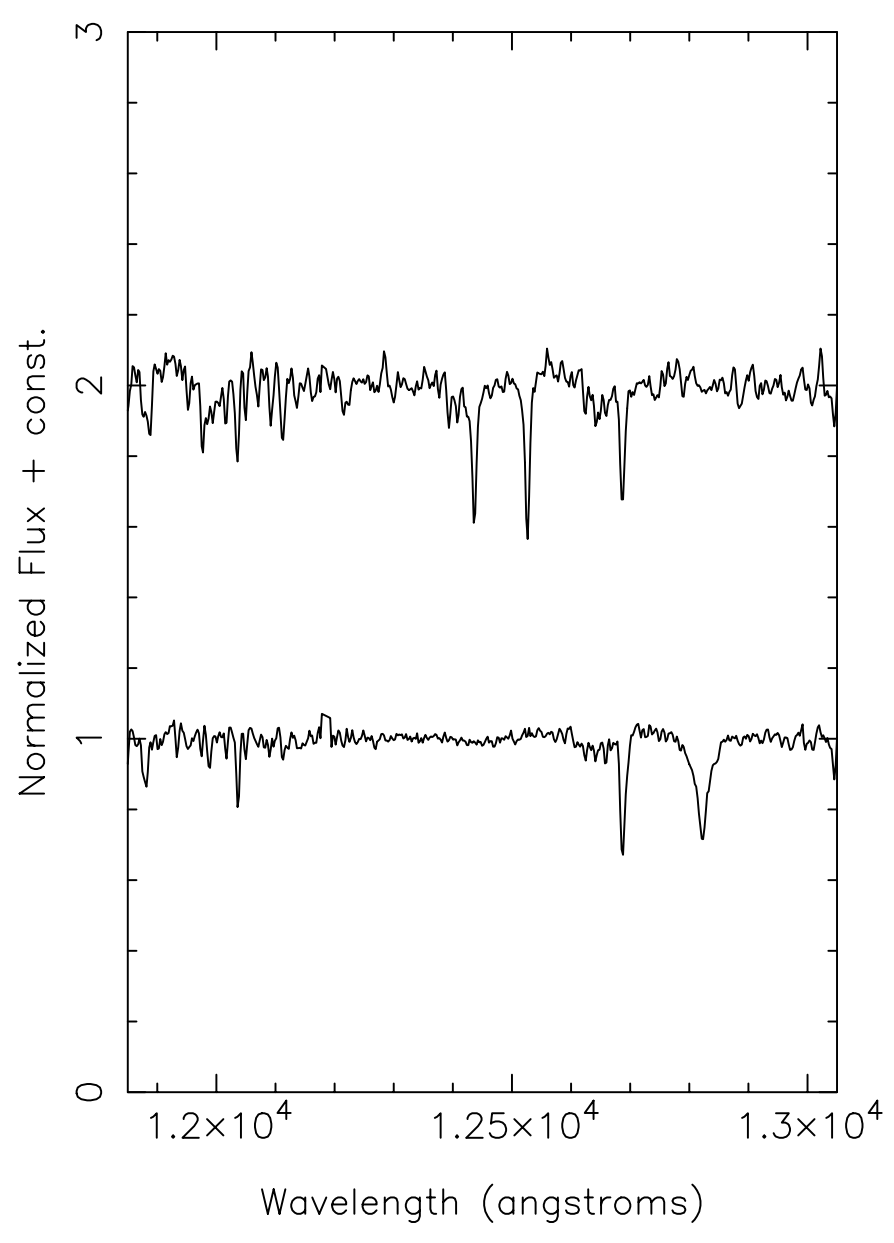

Fig. 5. The normalized spectrum of SIPS1259-4336 (top) along with that of an $\mathrm{A} 0$ standard star. The potassium doublet at 1.25 microns can clearly be seen.

obtained from IRIS2 at the Anglo-Australian Telescope. These data were reduced and the spectrum in the $J_{l}$ band is shown in Fig. 5. Using the equivalent width of the potassium doublet as a diagnostic (Gorlova et al. 2003) we find that it is likely to be an M 8 dwarf. The calculated parallax of SIPS1259-4336 of $276 \pm 41$ milliarcsec relates to a distance of $3.62 \pm 0.54$ parsecs. This is nearer than expected as while the object is of similar colour to SCR1845-6357 it is one magnitude dimmer, hence it should be much further away. However if SCR1845-6357 is an unresolved double then the distance quoted here could be accurate. (a)

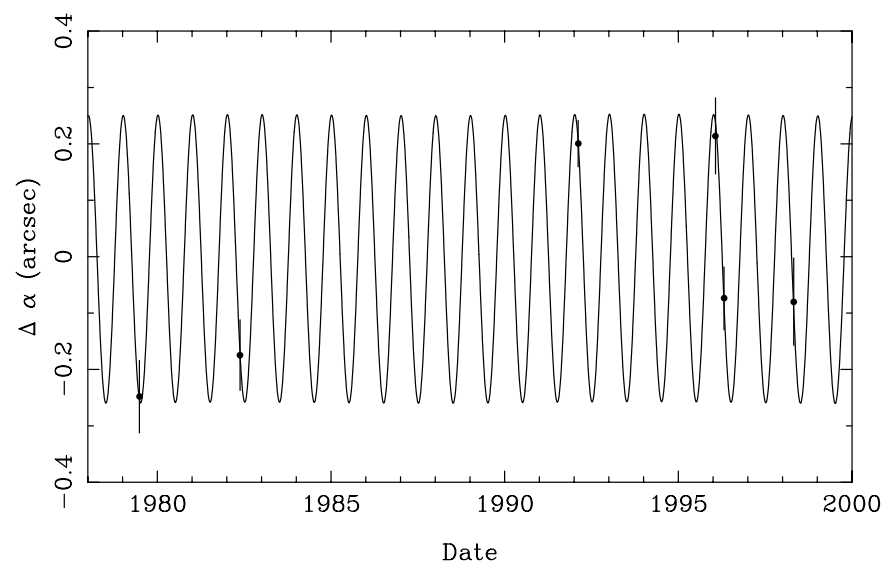

(b)

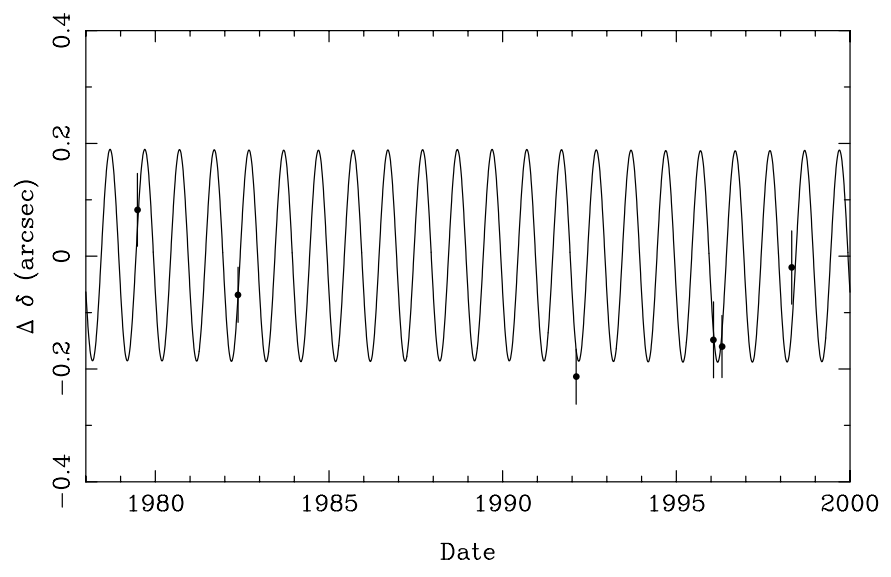

Fig. 6. The deviation from the proper motion in RA and Dec plotted against time. The line shown is the path of parallax motion predicted by the astrometric solution.

\subsubsection{SIPS1910-4132}

When the candidate image for SIPS1910-4232 was inspected it was found that there were two objects moving with a similar proper motion. Astrometric solutions for these companion objects were found by the $I$ plate-2MASS method as described in Sect. 3 and are shown in Table 3. It is clear that the three objects share a common proper motion and hence are likely to be a triple system. Table 4 shows the photometry for these objects. They all appear to be mid-late $\mathrm{M}$ dwarfs.

\subsubsection{SIPS0052-6201}

The astrometric solution for this object suggests that it has a common proper motion with LHS 128. LHS 128 is a K5 dwarf with a parallax of 52 mas (Perryman et al. 1997), if the two objects are part of the same system this yields a separation perpendicular to the line of sight of roughly $50000 \mathrm{AU}$. It is also possible that the two objects are not part of a binary system but are simply members of the same moving group. 


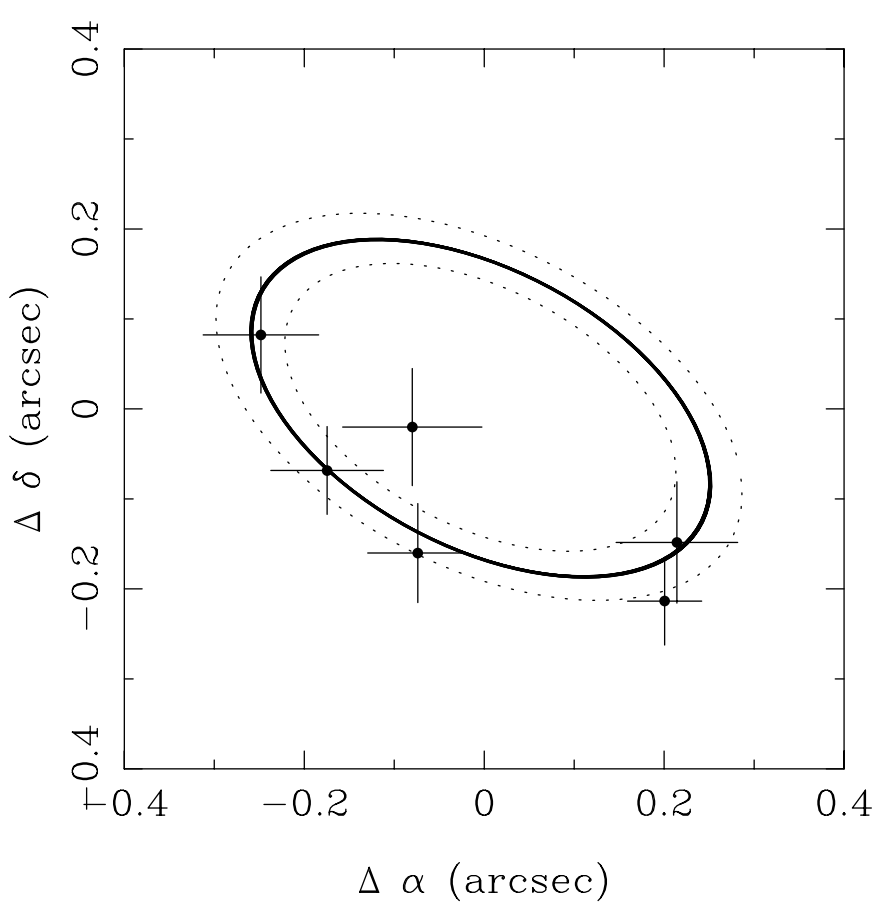

Fig. 7. The parallax ellipse traced out by SIPS1259-4336; the dotted lines represent one sigma upper and lower limits on the parallax.

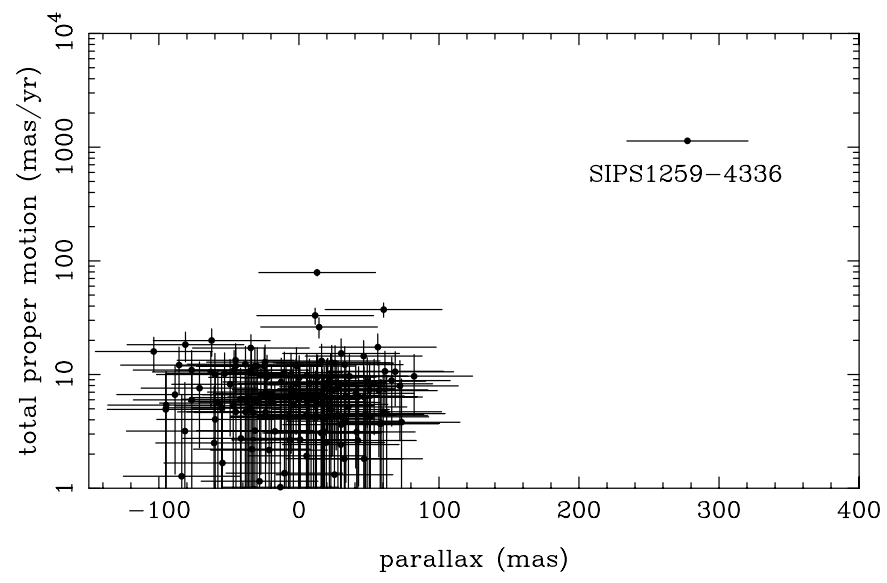

Fig. 8. Comparison of the fitted parallax and proper motions for the reference stars with SIPS1259-4336.

\section{Completeness}

\subsection{Areal completeness}

As the final aim of this survey is to produce an estimate for the local space density of late M, L and T Dwarfs we need to analyse the area covered by our survey. We have already excluded objects near the Galactic Plane due to crowding. Our other main problem related to crowding is that of the proximity flag given in the 2MASS Point Source Catalogue. As advised by the 2MASS Executive Summary we have excluded all objects within 6 arcsec of their nearest neighbour; this will present completeness problems in crowded regions. In order to produce an estimate as to the magnitude of these completeness problems we produced a series of simulations which allow us to calculate the probability of an object having a

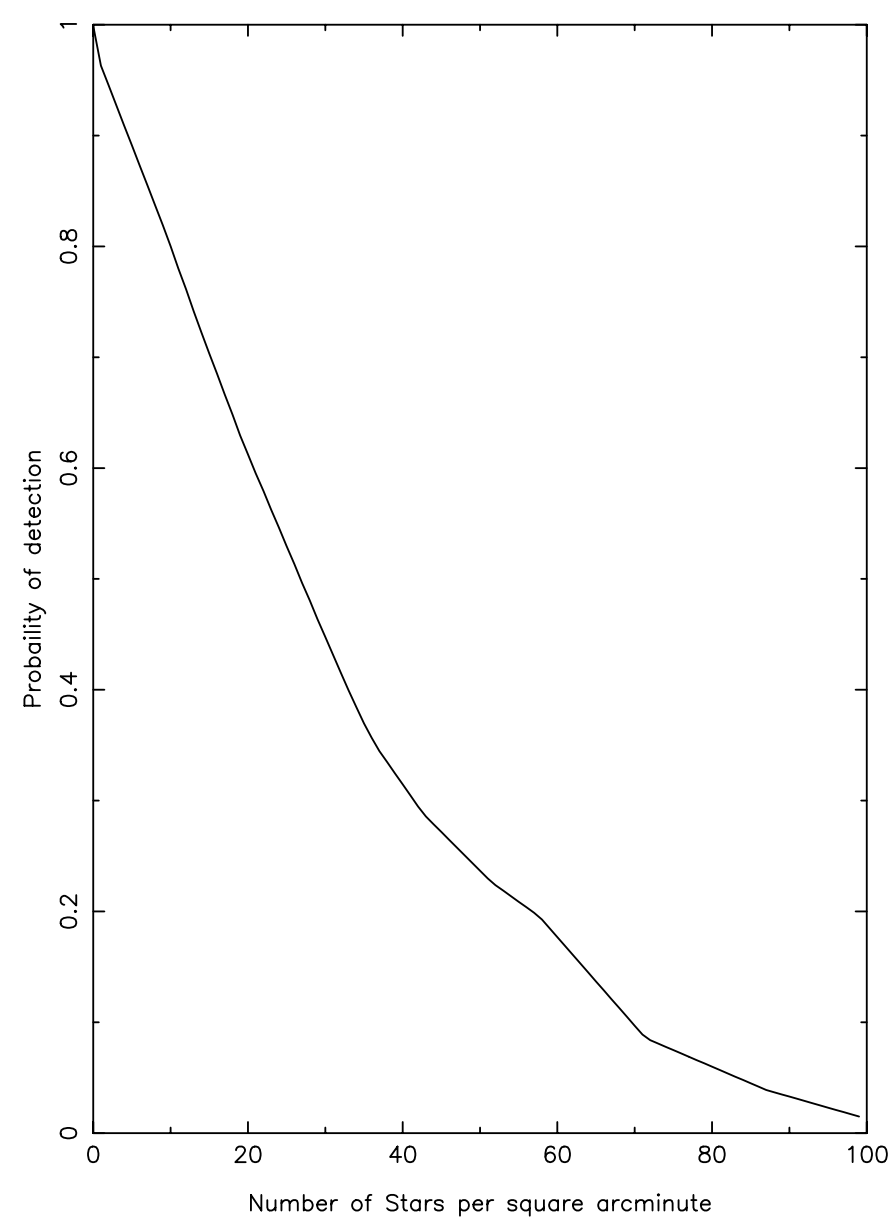

Fig. 9. The probability of detection plotted against the number of 2MASS images per square arc minute.

proximity flag greater than 6 arcsec for a given sky surface density of 2 MASS images. To do this a number of images were randomly placed in a one arcminute square box. If any of these fell with 6 arcsec of the centre the test was deemed to have failed. However if no image fell with 6 arcsec of the centre the the test was deemed to have succeeded. The probability of detection is thus the number of successful tests divided by the total number of tests. Simulations were carried out for different numbers of stars per square arc minute ranging from 1 to 100. The results of these simulations are shown in Fig. 9. In order to calculate the probability of objects being identified in particular regions of the sky, the number of 2MASS images per square arcminute and hence the probability of detection were calculated for each UKST field.

In addition there will be areal completeness problems caused by areas of UKST plates which are near to bright stars. Luckily the size of these areas is calculated in the post processing of each SuperCOSMOS scan. Hence we simply multiply this probability of detection that for the 2MASS images to yield an estimate of the areal completeness for each field. Figure 10 shows these data as a grey-scale plot. It is clear that the galactic plane is crowded and hence unsuitable for inclusion in this survey.

Further completeness issues will need to be addressed before a proper estimate of the the luminosity function (and hence 
Table 3.

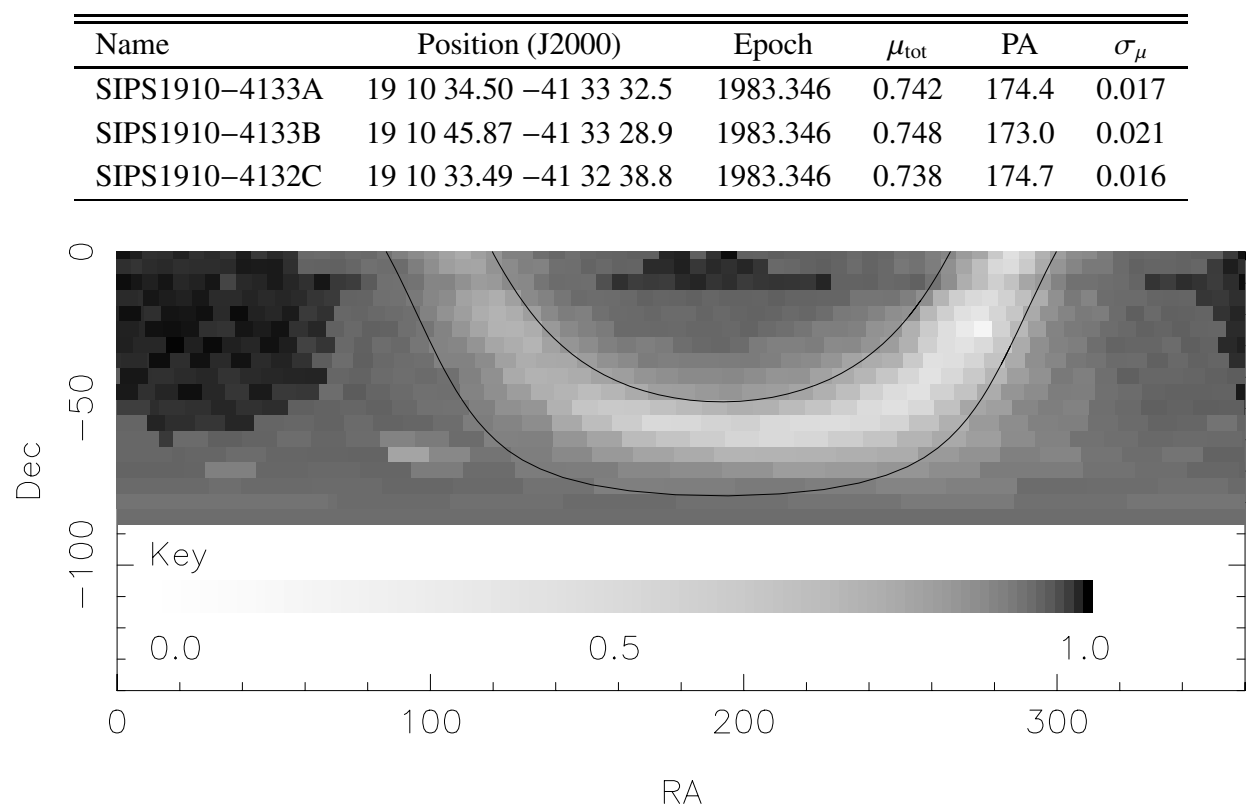

Fig. 10. The areal completeness of the survey. The scale shows the probability that an area is free from both crowding and bright stars. The solid lines marked represent the galactic latitude cut.

mass function) of the objects included here can be determined. Proper motion completeness will need to be simulated for, with the main factors being the one arcsecond "movement" cut and the lower proper motion limit. Also photometric completeness must be estimated taking both the 2MASS colour cut and the limit on the $I$ plate magnitude.

\subsection{Comparison with other studies}

In order to make clear the rough extent of the currently unquantified incompleteness due to astrometric and deblending cuts the survey was compared to the Luyten Half-Arcsecond Catalogue (Luyten 1979). Using the accurate positions of Bakos et al. (2002) the 2MASS data for the LHS objects was extracted. These objects were then subjected to the photometric, crowding and bright star cuts as the survey data. Figure 11 shows the number that could be detected and those that were. It is clear that the survey is somewhat incomplete, with only 43 of the 200 Luyten objects recovered. This is mainly due to many 2MASS and $I$ observations being taken at virtually the same time leading to a short proper motion baseline but deblended and elliptical objects will also play a role. However this is not an accurate estimate of the incompleteness of the survey. As the Luyten survey will be unquantifiably incomplete itself it will be necessary to produce simulations for both the photometric and astrometric incompleteness in order to gain a complete picture of the total incompleteness of the survey.

\section{Conclusion and further work}

We have utilised wide field surveys to discover 72 new objects with proper motions greater than $0.5^{\prime \prime} / \mathrm{yr}$. Of these objects we have discovered that one of them has a significant trigonometric
Table 4.

\begin{tabular}{lcccc}
\hline \hline Object & $I$ & $J$ & $H$ & $K_{\mathrm{S}}$ \\
\hline SIPS1910-4133A & 11.008 & 9.851 & 9.245 & 9.032 \\
SIPS1910-4133B & 11.738 & 10.61 & 10.002 & 9.739 \\
SIPS1910-4132C & 12.577 & 11.147 & 10.552 & 10.249 \\
\hline
\end{tabular}

parallax of $\pi=276 \pm 41$ milliarcsec yielding a distance of $3.62 \pm 0.54 \mathrm{pc}$.

In this paper our lower proper motion sets a bias towards nearer and hence brighter objects. In future papers we hope to detect objects with much lower proper motions allowing us to expand our search volume greatly. We will also attempt to identify ultracool members of different kinematic populations by means of reduced proper motions. Once the SuperCOSMOS Sky Survey is completed on the northern sky we hope to make use of these data too. Finally we hope to utilise the data yieled to these studies along with more substantial magnitude, proper motion and volume completeness estimates to calculate the local space density of late M, L and T dwarfs.

Acknowledgements. The authors would like to thank Sue Tritton and Mike Read for their help in selecting plates, Harvey MacGillivray and Eve Thomson for their prompt scanning of the material on SuperCOSMOS, David Bacon for his useful discussions on statistics, Mairi Brookes and Jessica Skelton for their discussion on spectroscopy and to Stuart Ryder for taking spectroscopic observations. This publication makes use of data products from the Two Micron All Sky Survey, which is a joint project of the University of Massachusetts and the Infrared Processing and Analysis Center/California Institute of Technology, funded by the National Aeronautics and Space Administration and the National Science Foundation. SuperCOSMOS is funded by a grant from the UK Particle Physics and Astronomy Research Council. 

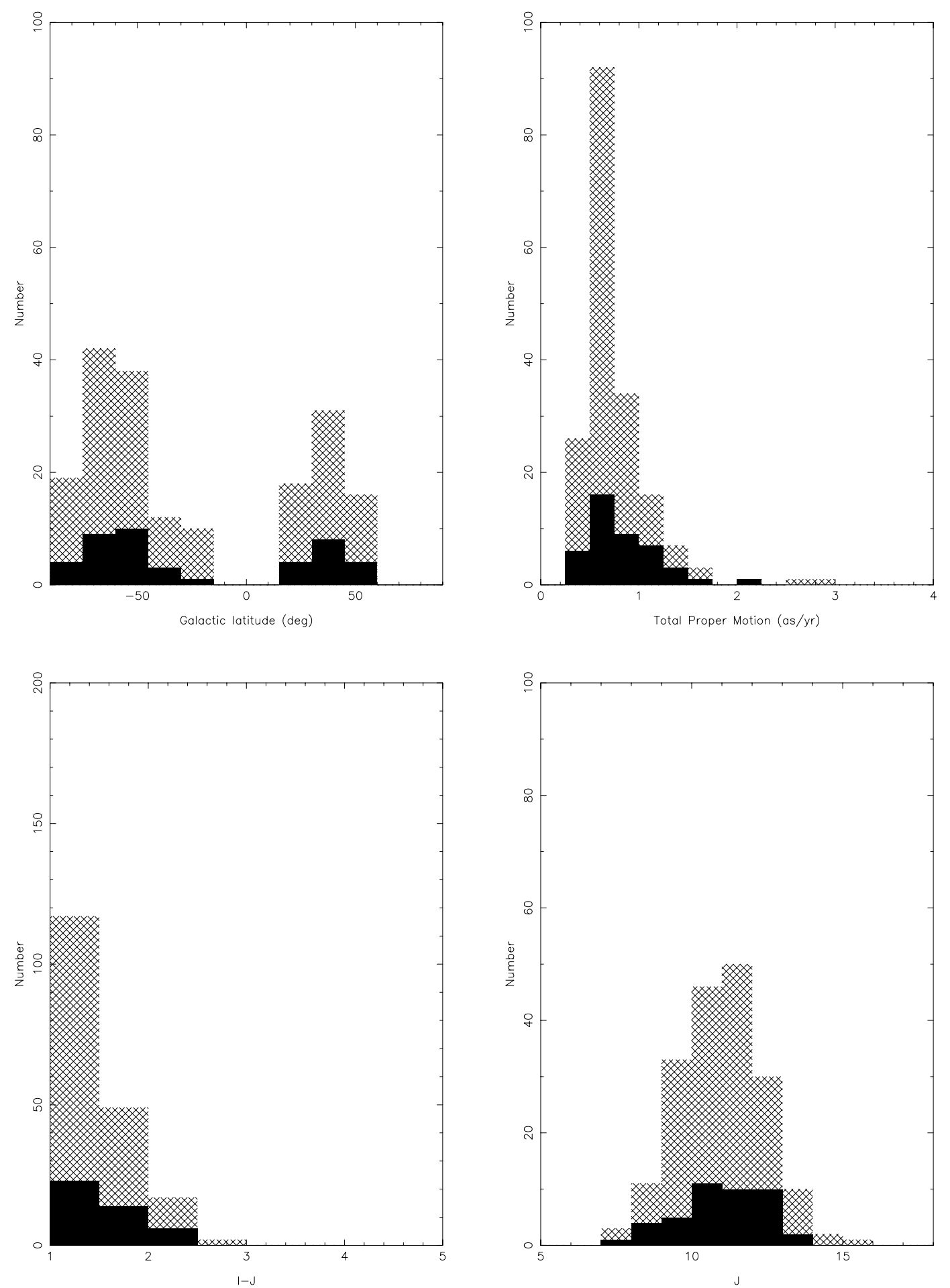

Fig. 11. The number of Luyten objects that could possibly be detected (grey) and those that were (solid).

\section{References}

Bakos, G., Sahu, K. C., \& Nemeth, P. 2002, ApJS, 141, 187

Burgasser, A. J., Kirkpatrick, J. D., Brown, M. E., et al. 2002, ApJ, 564,421

Burgasser, A. J., McElwain, M. W., Kirkpatrick, J. D., et al. 2004, AJ, 127,2856

Dahn, C. C., Harris, H. C., Vrba, F. J., et al. 2002, AJ, 124, 1170

Deacon, N. R., \& Hambly, N. C. 2001, A\&A, 380, 148

Deacon, N. R., Hambly, N. C., Henry, T. J., et al. 2005, AJ, 129, 409
Geballe, T. R., Knapp, G. R., Leggett, S. K., et al. 2002, ApJ, 564, 466 Gorlova, N. I., Meyer, M. R., Rieke, G. H., \& Liebert, J. 2003, ApJ, 593, 1074

Hambly, N. C., Davenhall, A. C., Irwin, M. J., \& MacGillivray, H. T. 2001, MNRAS, 326, 1315

Hambly, N. C., Henry, T. J., Subasavage, J., Brown, M., \& Jao, W. C. 2004, AJ, 128, 437

Kendall, T. R., Delfosse, X., Martin, E. L., \& Forveille, T. 2004, A\&A 416, L17

Kirkpatrick, J. D., Reid, I. N., Liebert, J., et al. 2000, AJ, 120, 447 
Lepine, S., \& Shara, M. M. 2002, AJ, 124

Luyten, W. J. 1979, Luyten Half Arcsecond Catalogue, University of Minnesota, Minneaplois

Perryman, M. A. C., Lindegren, L., Kovalevsky, J., et al. 1997, A\&A, 323, L49

Pokorny, R. S., Jones, H. R. A., \& Hambly, N. C. 2003, A\&A, 397, 575

Ruiz, M. T., Wischnjewsky, M., Rojo, P. M., \& Gonzalez, L. E. 2001, ApJSS, 133, 119

Subasavage, J. P., Henry, T. J., Hambly, N. C., Brown, M. A., \& Jao, W. C. 2005, AJ, submitted

Scholz, R. D., Ibata, R., Irwin, M., et al. 2002, MNRAS, 329, 109
Scholz, R. D., McCaughrean, M. J., Lodieu, N., \& Kuhlbrodt, B. 2004, A\&A, 398, L29

Seifahrt, A., Neuhäuser, R., \& Mugrauer, M. 2004, A\&A, 421, 255

Teegarden, B. J., Pravdo, S. H., Hicks, M., et al. 2003, ApJ, 589, L51

Vbra, F. J., Henden, A. A., Luginbuhl, C. B., et al. 2004, AJ, 127, 2948

Wallace, P. T. 1998, Starlink User Note No. 67.42: SLALIB:

Postitional Astronomy Library, CCLRC/Rutherford Appleton Laboratory, PPARC

Wroblewski, H., \& Costa, E. 2001, A\&A, 367, 725

The 2MASS Point Source Catalogue Executive Summary, http://pegasus. phast. umass.edu/ 